\title{
Efeito da substituição da gordura suína por óleos vegetais nas características de qualidade, estabilidade oxidativa e microestrutura de mortadela
}

\section{Effect of pork backfat replacement for vegetal oils in the quality characteristics, oxidative stability and microstructure of mortadella}

\author{
João Felipe Ferraz Yunes ${ }^{1}$; Carlos Pasqualin Cavalheiro'; \\ Liana Inês Guidolin Milani'; Marina Bergoli Scheeren ${ }^{1}$; \\ Francisco Javier Hernandez Blazquez²; Cristiano Augusto Ballus ${ }^{3}$; \\ Leadir Lucy Martins Fries ${ }^{4}$; Nelcindo Nascimento Terra ${ }^{4 *}$
}

\section{Resumo}

\begin{abstract}
Este trabalho foi desenvolvido com o objetivo de avaliar o uso de diferentes óleos vegetais como substituto da gordura animal em mortadela. Para isso, foram utilizados os óleos de canola, linhaça, oliva e soja em dois níveis de substituição (50 e 100\%). Foram avaliadas as características físico-químicas (composição centesimal, pH e Atividade de água), cor instrumental, características microbiológicas, oxidação lipídica (TBARS) e microestrutura durante armazenamento em temperatura ambiente por 60 dias. Os produtos apresentaram os parâmetros de qualidade exigidos pela legislação brasileira. Houve uma queda nos valores de $\mathrm{pH}$ durante o armazenamento devido ao crescimento de bactérias láticas. Os produtos contendo óleos vegetais apresentaram teores de luminosidade $\left(L^{*}\right)$ e amarelo $\left(b^{*}\right)$ superiores e teor de vermelho $\left(a^{*}\right)$ inferior ao controle. Os valores de TBARS mantiveram-se abaixo do limiar perceptível pelo consumidor e as características de microestrutura foram semelhantes às observadas em outros produtos cárneos cozidos. Assim, a utilização de óleos de canola, linhaça, oliva ou soja é uma alternativa viável como substituto da gordura animal na elaboração de mortadela.

Palavras-chave: Gordura suína, óleos vegetais, oxidação lipídica, microestrutura, mortadela
\end{abstract}

\begin{abstract}
This study was developed with the aim to evaluate the use of different vegetable oils as a replacer for pork backfat. For this, there were used canola, linseed, olive and soybean oils at two levels of replacement (50 and 100\%). There were evaluated the physico-chemical characteristics (proximate composition, $\mathrm{pH}$ and water activity), instrumental color, microbiological characteristics, lipid oxidation (TBARS) and microstructure during storage at room temperature for 60 days. The products had the quality parameters required by the Brazilian legislation. There was a $\mathrm{pH}$ decrease during storage due to the growth of lactic
\end{abstract}

1 Discente(s) do Programa de Pós-Graduação em Ciência e Tecnologia de Alimentos, Universidade Federal de Santa Maria, UFSM, Santa Maria, RS. E-mail: joao.yunes@hotmail.com; cavalheiro.carlos@hotmail.com; lianamilani@yahoo.com.br; marinabergoli@hotmail.com;

2 Prof. da Faculdade de Medicina Veterinária e Zootecnia, Departamento de Cirurgia, Universidade de São Paulo, USP, São Paulo, SP. E-mail: fjhblazq@usp.br

3 Discente do Programa de Pós-Graduação em Ciência de Alimentos, Universidade Estadual de Campinas, UNICAMP, Campinas, SP. E-mail: cristiano_ballus@yahoo.com.br

4 Profs. do Programa de Pós-Graduação em Ciência e Tecnologia de Alimentos, UFSM, Santa Maria, RS. E-mail: lucymicro@ yahoo.com.br; nelcindo@terra.com.br

Autor para correspondência 
acid bacteria. Products containing vegetable oils showed higher levels of lightness $\left(L^{*}\right)$ and yellowness $\left(b^{*}\right)$ and lower levels of redness $\left(a^{*}\right)$. TBARS values remained under the perceptible threshold by the consumers and microstructure characteristics were similar to those observed in other kinds of cooked meat sausages. Thus, the use of canola, linseed, olive or soybean oils is a viable alternative as a substitute for pork backfat in elaboration of mortadella.

Key words: Pork backfat, vegetal oils, lipid oxidation, microstructure, mortadella

\section{Introdução}

Recentemente houve um crescimento na demanda por produtos cárneos mais saudáveis. A formulação deste tipo de produto é baseada nas estratégias de processamento como uma das formas de desenvolvimento de potenciais produtos funcionais a base de carne. Numerosos ingredientes não cárneos têm sido usados para reformular produtos cárneos e, assim incluir compostos mais saudáveis na sua formulação (MUGUERZA; ANSORENA; ASTIASARÁN, 2004; FERNÁNDEZ-GINÉS et al., 2005; ARIHARA, 2006; JIMÉNEZCOLMENERO; REIG; TOLDRÁ, 2006).

Por causa da sua importância, os lipídeos estão entre os compostos bioativos (ingredientes funcionais) que tem recebido maior atenção no que diz respeito ao desenvolvimento de produtos cárneos saudáveis (JIMÉNEZ-COLMENERO, 2007). Para melhorar o perfil lipídico geralmente se faz a substituição da gordura animal presente normalmente no produto por outra gordura que tenha características mais favoráveis.

Nesse sentido, diversos estudos já foram realizados nos mais diferentes produtos como linguiças fermentadas (MUGUERZA et al., 2002; HOZ et al., 2004; PELSER et al., 2007), salame italiano (DEL NOBILE et al., 2009), presunto curado e seco (SANTOS et al., 2008), "Chorizo de Pamplona" (MUGUERZA et al., 2001; MUGUERZA; ANSORENA; ASTIASARÁN, 2003), linguiças frescais (VALENCIA et al., 2008) e salsichas (LURUEÑA-MARTÍNEZ; VIVARQUINTANA; REVILLA, 2004). No entanto, ainda são escassos estudos em relação à substituição de gordura animal em produtos cozidos, especialmente aqueles conservados sob temperatura ambiente.
Portanto, este trabalho teve por objetivo avaliar o efeito da substituição da gordura suína, em dois níveis (50 e 100\%), nas características de qualidade (propriedades físico-químicas, cor instrumental e características microbiológicas), estabilidade oxidativa (TBARS) e microestrutura de mortadelas elaboradas com óleos vegetais de canola, linhaça, oliva ou soja.

\section{Materiais e Métodos}

Os tratamentos foram codificados da seguinte maneira: P, C1, C2, L1, L2, O1, O2, S1 e S2, onde as letras indicam o tipo de óleo utilizado sendo "C" para canola; "L" para linhaça; "O" para oliva e "S" para soja e os algarismos indicam o nível de substituição, sendo " 1 " para $50 \%$ de substituição da gordura suína e “2” para $100 \%$. A letra P foi adotada para o tratamento controle.

A fabricação das mortadelas foi feita em um frigorífico localizado em Santa Catarina, sob Sistema de Inspeção Federal (SIF), utilizando formulação e procedimento usuais propostos por Terra (1998). Primeiramente as matérias-primas foram cominuídas em moedor modelo 200 (Tetra Laval Food ${ }^{\circledR}$, Pully, Suíça) com disco de $8 \mathrm{~mm}$. Após, a carne bovina $(75,00 \%)$ e a emulsão de couro suíno $(5,00 \%)$ foram levadas ao cutter modelo K324 K-U (Seydelmann ${ }^{\circledR}$, Aalen, Alemanha), juntamente com o sal $(1,90 \%)$ e o polifosfato de sódio $(0,25 \%)$. Após homogeneização, foi adicionado o restante dos ingredientes: gelo $(29,00 \%)$, proteína de soja $(2,00 \%)$, nitrito de sódio $(0,016 \%)$, condimento para mortadela $(1,00 \%)$, carragena $(0,50 \%)$, alho natural $(0,12 \%)$ e pimenta semi moída $(0,10 \%)$. Então, foi adicionado o toucinho na quantidade de $15,00 \%$ para o tratamento controle, $7,50 \%$ 
de óleo vegetal mais $7,50 \%$ de toucinho nos tratamento com substituição de $50 \%$ e $15,00 \%$ de óleo vegetal para os tratamentos com 100\% de substituição do toucinho por óleo vegetal. Após, a mistura foi homogeneizada sob vácuo e embutida em embutideira modelo VF630 (Handtmann ${ }^{\circledR}$, Biberach an der Riss, Alemanha) também sob vácuo em tripas celulósicas, perfazendo peças de $5 \mathrm{~kg}$. O cozimento foi feito em etapas alternadas de secagem e defumação, até que o centro geométrico do produto atingisse temperatura de $73{ }^{\circ} \mathrm{C}$. Finalizado o cozimento, as peças foram resfriadas até atingir $22{ }^{\circ} \mathrm{C}$. Por fim, as peças foram seladas a vácuo e armazenadas em temperatura entre $19{ }^{\circ} \mathrm{C}$ e $25^{\circ} \mathrm{C}$.

As análises de composição centesimal foram realizadas conforme metodologias propostas por Terra e Brum (1988). Também foram realizadas análises de $\mathrm{pH}$ e Atividade de Água (Aa) em pHmêtro digital modelo DM20 (Digimed, São Paulo, Brasil) após homogeneização em água destilada e medidor específico de atividade de água modelo AquaLab CX2 (Decagon Devices Inc. ${ }^{\circledR}$, Pullman, Estados Unidos), respectivamente. Para a análise de cor instrumental, utilizou-se colorímetro Minolta CR400 (Minolta Chroma Meter ${ }^{\circledR}$, Osaka, Japão), calibrado previamente, operando com iluminante D-65 (MACDOUGALL, 2000) e ângulo de visão de $10^{\circ}$, expressando no sistema CIELAB os valores de luminosidade $\left(L^{*}\right)$, teor de vermelho $\left(a^{*}\right)$ e teor de amarelo $\left(b^{*}\right)$. A composição centesimal e a análise de cor instrumental foram realizadas apenas após a fabricação do produto.

Foram realizadas também contagem de microrganismos mesófilos aeróbios e bactérias ácido-láticas, conforme estabelecido pela legislação (BRASIL, 2003), utilizando os ágars PCA (Plate Count Agar) (Himedia Ltd., Mumbai, Índia) e MRS (De Man, Rogosa and Sharpe) (Himedia Ltd., Mumbai, Índia), com incubação a $37^{\circ} \mathrm{C}$ por 48 horas e $30{ }^{\circ} \mathrm{C}$ por 48 horas, respectivamente. Também foram medidas as substâncias reativas ao ácido tiobarbitúrico (TBARS) resultantes da oxidação lipídica conforme metodologia proposta por Raharjo, Sofos e Schmidt (1992), nos dias 0, 30 e 60 dias de armazenamento.

Por fim, a análise de microestrutura das mortadelas foi realizada em microscópio ótico binocular, na objetiva 10x, modelo BX 60 (Olympus Optical, São Paulo, Brasil), em amostras preparadas conforme procedimento descrito por Bancroft e Gamble (2002).

Todas as análises físico-químicas e microbiológicas foram feitas em triplicata, as análises de cor instrumental em sextuplicata e as de TBARS em duplicata. Os dados foram analisados por análise de variância (ANOVA), com delineamento inteiramente casualizado. As médias foram comparadas por teste de Tukey, com nível de significância de 5\% $(\mathrm{p}<0,05)$, utilizando o software estatístico SPSS 17.0.

\section{Resultados e Discussão}

Os valores de composição centesimal da mortadela (Tabela 1) apresentaram-se de acordo com a legislação vigente que determina o Padrão de Identidade e Qualidade de Mortadelas (BRASIL, 2000). Não foram observadas diferenças significativas entre os tratamentos nos parâmetros umidade, carboidratos e gordura. No entanto, foi possível observar que os tratamentos contendo $100 \%$ de óleo de linhaça (L2) e soja (S2) apresentaram teores de proteínas mais elevados. Esses teores elevados provavelmente sejam devido à variações nos cortes cárneos utilizados na fabricação das mortadelas. Singh et al. (2011) adicionaram até 4\% de óleo de linhaça em patê de frango e também não encontraram alterações na composição centesimal do produto. $\mathrm{O} \mathrm{pH}$ das amostras, no dia 0 , variou entre 5,92 a 6,61 (Tabela 2), sendo possível observar que o tratamento contendo $100 \%$ de óleo de canola (C2) apresentou o menor $\mathrm{pH}$ de todos os tratamentos. Ainda, houve uma redução nos valores de $\mathrm{pH}$ para todos os tratamentos durante o armazenamento. Essa queda é devido principalmente ao crescimento gradual de bactérias láticas, que produzem ácido 
lático que é responsável pela queda do $\mathrm{pH}$. Já em relação à Aa, não houve diferenças entre os tratamentos no dia 0 e no dia 60 , sendo os valores normais para o produto em questão. Os resultados referentes às características físico-químicas encontradas neste trabalho concordam com os obtidos por Bloukas e Paneras (1993), GrigelmoMiguel, Abadías-Serós e Martín-Belloso (1999), Cáceres et al. (2004), Cáceres, García e Selgas (2008) e Viuda-Martos et al. (2010).

Tabela 1. Composição centesimal de mortadelas elaboradas com diferentes óleos vegetais em dois níveis de substituição da gordura suína.

\begin{tabular}{ccccc}
\hline Tratamentos & Umidade & Carboidratos & Gordura & Proteína \\
\hline P & $58,23 \pm 0,82^{\mathrm{a}}$ & $6,32 \pm 0,46^{\mathrm{a}}$ & $16,19 \pm 2,28^{\mathrm{a}}$ & $12,61 \pm 1,38^{\mathrm{b}}$ \\
C1 & $60,39 \pm 0,37^{\mathrm{a}}$ & $7,08 \pm 0,61^{\mathrm{a}}$ & $13,81 \pm 1,49^{\mathrm{a}}$ & $13,02 \pm 0,27^{\mathrm{b}}$ \\
C2 & $59,75 \pm 0,57^{\mathrm{a}}$ & $6,30 \pm 0,23^{\mathrm{a}}$ & $12,72 \pm 0,73^{\mathrm{a}}$ & $13,06 \pm 0,09^{\mathrm{ab}}$ \\
L1 & $60,11 \pm 1,27^{\mathrm{a}}$ & $6,47 \pm 0,20^{\mathrm{a}}$ & $14,13 \pm 0,92^{\mathrm{a}}$ & $12,76 \pm 0,11^{\mathrm{b}}$ \\
L2 & $58,82 \pm 0,72^{\mathrm{a}}$ & $6,50 \pm 0,49^{\mathrm{a}}$ & $15,46 \pm 1,15^{\mathrm{a}}$ & $13,35 \pm 0,48^{\mathrm{a}}$ \\
O1 & $59,59 \pm 1,00^{\mathrm{a}}$ & $6,88 \pm 0,67^{\mathrm{a}}$ & $13,31 \pm 1,17^{\mathrm{a}}$ & $12,84 \pm 0,26^{\mathrm{b}}$ \\
O2 & $59,60 \pm 0,34^{\mathrm{a}}$ & $6,77 \pm 0,87^{\mathrm{a}}$ & $12,51 \pm 0,99^{\mathrm{a}}$ & $12,75 \pm 0,09^{\mathrm{b}}$ \\
S1 & $59,29 \pm 0,91^{\mathrm{a}}$ & $6,90 \pm 0,48^{\mathrm{a}}$ & $14,18 \pm 0,83^{\mathrm{a}}$ & $12,63 \pm 0,07^{\mathrm{b}}$ \\
S2 & $59,45 \pm 0,84^{\mathrm{a}}$ & $6,61 \pm 0,28^{\mathrm{a}}$ & $14,96 \pm 1,42^{\mathrm{a}}$ & $14,68 \pm 0,79^{\mathrm{a}}$ \\
\hline
\end{tabular}

${ }^{a}$ Médias acompanhadas pela mesma letra na mesma coluna, não apresentam diferença significativa pelo teste de Tukey.

P - 100\% de gordura suína; C1 - 50\% de óleo de canola; C2 - 100\% de óleo de canola; L1 - 50\% de óleo de linhaça; L2 - 100\% de óleo de linhaça; O1 - 50\% de óleo de oliva; O2 - 100\% de óleo de oliva; S1 - 50\% de óleo de soja; S2 - 100\% de óleo de soja. Fonte: Elaboração dos autores.

Tabela 2. pH e Atividade de água (Aa) de mortadelas elaboradas com diferentes óleos vegetais em dois níveis de substituição da gordura suína durante armazenamento por 60 dias em temperatura ambiente.

\begin{tabular}{ccccccc}
\hline \multirow{2}{*}{ Tratamentos } & \multicolumn{5}{c}{ pH } & \multicolumn{3}{c}{ Aa } \\
\cline { 2 - 7 } & Dia 0 & Dia 30 & Dia 60 & Dia 0 & Dia 30 & Dia 60 \\
\hline P & $6,33 \pm 0,01^{\mathrm{d}}$ & $6,18 \pm 0,02^{\mathrm{a}}$ & $5,81 \pm 0,01^{\mathrm{c}}$ & $0,960 \pm 0,003^{\mathrm{a}}$ & $0,970 \pm 0,003^{\mathrm{a}}$ & $0,965 \pm 0,001^{\mathrm{ab}}$ \\
$\mathrm{C} 1$ & $6,50 \pm 0,01^{\mathrm{c}}$ & $6,04 \pm 0,00^{\mathrm{d}}$ & $5,65 \pm 0,01^{\mathrm{e}}$ & $0,960 \pm 0,002^{\mathrm{a}}$ & $0,963 \pm 0,001^{\mathrm{b}}$ & $0,959 \pm 0,005^{\mathrm{b}}$ \\
$\mathrm{C} 2$ & $5,92 \pm 0,02^{\mathrm{e}}$ & $5,96 \pm 0,00^{\mathrm{d}}$ & $5,72 \pm 0,01^{\mathrm{d}}$ & $0,956 \pm 0,003^{\mathrm{a}}$ & $0,962 \pm 0,004^{\mathrm{a}}$ & $0,956 \pm 0,002^{\mathrm{b}}$ \\
L1 & $6,53 \pm 0,01^{\mathrm{bc}}$ & $6,09 \pm 0,01^{\mathrm{c}}$ & $5,83 \pm 0,01^{\mathrm{c}}$ & $0,961 \pm 0,003^{\mathrm{a}}$ & $0,967 \pm 0,002^{\mathrm{ab}}$ & $0,963 \pm 0,003^{\mathrm{ab}}$ \\
L2 & $6,61 \pm 0,03^{\mathrm{a}}$ & $6,06 \pm 0,02^{\mathrm{d}}$ & $5,90 \pm 0,00^{\mathrm{b}}$ & $0,962 \pm 0,002^{\mathrm{a}}$ & $0,968 \pm 0,002^{\mathrm{ab}}$ & $0,961 \pm 0,002^{\mathrm{ab}}$ \\
O1 & $6,49 \pm 0,02^{\mathrm{c}}$ & $6,12 \pm 0,01^{\mathrm{b}}$ & $5,45 \pm 0,03^{\mathrm{f}}$ & $0,959 \pm 0,005^{\mathrm{a}}$ & $0,967 \pm 0,004^{\mathrm{ab}}$ & $0,958 \pm 0,001^{\mathrm{ab}}$ \\
O2 & $6,50 \pm 0,00^{\mathrm{c}}$ & $6,04 \pm 0,00^{\mathrm{d}}$ & $5,65 \pm 0,00^{\mathrm{e}}$ & $0,954 \pm 0,003^{\mathrm{a}}$ & $0,966 \pm 0,001^{\mathrm{a}}$ & $0,961 \pm 0,000^{\mathrm{ab}}$ \\
S1 & $6,53 \pm 0,01^{\mathrm{bc}}$ & $6,09 \pm 0,00^{\mathrm{c}}$ & $6,01 \pm 0,01^{\mathrm{a}}$ & $0,959 \pm 0,003^{\mathrm{a}}$ & $0,966 \pm 0,003^{\mathrm{ab}}$ & $0,961 \pm 0,002^{\mathrm{ab}}$ \\
S2 & $6,54 \pm 0,01^{\mathrm{b}}$ & $6,10 \pm 0,01^{\mathrm{c}}$ & $5,97 \pm 0,03^{\mathrm{a}}$ & $0,955 \pm 0,003^{\mathrm{a}}$ & $0,963 \pm 0,002^{\mathrm{a}}$ & $0,960 \pm 0,002^{\mathrm{ab}}$ \\
\hline
\end{tabular}

a Médias acompanhadas pela mesma letra na mesma coluna, não apresentam diferença significativa pelo teste de Tukey. P - 100\% de gordura suína; C1 - 50\% de óleo de canola; C2 - 100\% de óleo de canola; L1 - 50\% de óleo de linhaça; L2 - 100\% de óleo de linhaça; O1 - 50\% de óleo de oliva; O2 - 100\% de óleo de oliva; S1 - 50\% de óleo de soja; S2 - 100\% de óleo de soja. Fonte: Elaboração dos autores. 
A mensuração da cor é um importante parâmetro a ser analisado em produtos cozidos, pois os consumidores associam a este produto a característica de coloração rosa e brilhante (CÁCERES, GARCÍA; SELGAS, 2006). As mudanças observadas nos parâmetros de cor são basicamente relacionadas com a diminuição no teor de gordura. Alguns autores (CLAUS; HUNT; KASTNER, 1989; PANERAS; BLOUKAS; PAPADIMA, 1996) já relataram que quando o conteúdo de gordura é reduzido, os valores de $L^{*}$ e $b^{*}$ reduzem, enquanto que os valores de $a^{*}$ aumentam. No entanto, em outros estudos já realizados não foram observadas diferenças nos parâmetros de cor em relação à alterações no teor de gordura (BARBUT; MITTAL, 1995). A luminosidade ( $\left.L^{*}\right)$ em alimentos é afetada por diversos fatores, incluindo a concentração e o tipo de pigmentos presentes (LINDAHL;
LUNDSTRÖM; TORNBERG, 2001), umidade (ALESON-CARBONELL et al., 2002) e o conteúdo e o tipo de fibras (FERNÁNDEZ-GINÉS et al., 2003). Uma redução nos teores de gordura pode produzir produtos mais escuros (teores de luminosidade menores), como os já encontrados por Cáceres et al. (2004) e Cáceres, García e Selgas (2006) em mortadela e por Claus e Hunt (1991), Troutt et al. (1992) e Grigelmo-Miguel, AbadíasSerós e Martín-Belloso (1999) em outros produtos cozidos. No entanto, a substituição de 100\% da gordura animal por óleo de canola produziu mortadelas com teores de luminosidade superiores (Tabela 3). Isso pode ser explicado pelo fato do óleo de canola possuir glóbulos de gordura menores, mas em maior quantidade que refletem mais a luz do que os glóbulos maiores dos outros óleos utilizados e da própria carne (YOUSSEF; BARBUT, 2011).

Tabela 3. Valores médios dos parâmetros de cor instrumental e do parâmetro de textura dureza de mortadelas elaboradas com diferentes óleos vegetais em dois níveis de substituição da gordura suína.

\begin{tabular}{cccc}
\hline \multirow{2}{*}{ Tratamentos } & \multicolumn{3}{c}{ Parâmetros de cor instrumental } \\
\cline { 2 - 4 } & $\boldsymbol{L}^{*}$ & $\boldsymbol{a}^{*}$ & $\boldsymbol{b}^{*}$ \\
\hline $\mathrm{P}$ & $53,94 \pm 0,36^{\mathrm{c}}$ & $18,03 \pm 0,12^{\mathrm{a}}$ & $9,49 \pm 0,58^{\mathrm{d}}$ \\
$\mathrm{C} 1$ & $58,53 \pm 1,85^{\mathrm{ab}}$ & $15,68 \pm 0,13^{\mathrm{c}}$ & $10,42 \pm 0,47^{\mathrm{bc}}$ \\
$\mathrm{C} 2$ & $58,66 \pm 0,63^{\mathrm{a}}$ & $15,90 \pm 0,29^{\mathrm{c}}$ & $10,35 \pm 0,44^{\mathrm{bc}}$ \\
$\mathrm{L} 1$ & $54,09 \pm 1,32^{\mathrm{c}}$ & $16,94 \pm 0,39^{\mathrm{b}}$ & $10,92 \pm 0,45^{\mathrm{ab}}$ \\
$\mathrm{L} 2$ & $55,58 \pm 1,11^{\mathrm{bc}}$ & $16,93 \pm 0,45^{\mathrm{b}}$ & $10,72 \pm 0,48^{\mathrm{ab}}$ \\
$\mathrm{O} 1$ & $55,77 \pm 1,95^{\mathrm{bc}}$ & $16,14 \pm 0,87^{\mathrm{bc}}$ & $11,11 \pm 0,23^{\mathrm{a}}$ \\
O2 & $58,17 \pm 1,50^{\mathrm{ab}}$ & $15,92 \pm 0,60^{\mathrm{c}}$ & $10,75 \pm 0,05^{\mathrm{ab}}$ \\
$\mathrm{S} 1$ & $51,11 \pm 2,47^{\mathrm{c}}$ & $16,97 \pm 0,87^{\mathrm{c}}$ & $10,02 \pm 0,53^{\mathrm{cd}}$ \\
$\mathrm{S} 2$ & $56,40 \pm 1,87^{\mathrm{bc}}$ & $16,54 \pm 0,34^{\mathrm{bc}}$ & $10,79 \pm 0,21^{\mathrm{ab}}$ \\
\hline
\end{tabular}

a Médias acompanhadas pela mesma letra na mesma coluna, não apresentam diferença significativa pelo teste de Tukey. P - 100\% de gordura suína; C1 - 50\% de óleo de canola; C2 - 100\% de óleo de canola; L1 - 50\% de óleo de linhaça; L2 - 100\% de óleo de linhaça; O1 - 50\% de óleo de oliva; O2 - 100\% de óleo de oliva; S1 - 50\% de óleo de soja; S2 - 100\% de óleo de soja. Fonte: Elaboração dos autores.

Os teores de vermelho $\left(a^{*}\right)$ e amarelo $\left(b^{*}\right)$ apresentam um comportamento similar à luminosidade $\left(L^{*}\right)$. Em relação ao teor de vermelho $\left(a^{*}\right)$, foi possível observar que a diferença se baseou nos tipos de óleos adicionados e não nas suas quantidades. Porém, o controle apresentou os maiores teores de vermelho (Tabela 3), mostrando que a substituição da gordura animal por óleos vegetais altera esta característica em mortadelas. $\mathrm{O}$ teor de amarelo $\left(b^{*}\right)$ também apresentou diferenças significativas entre os tratamentos, sendo possível observar que o tratamento controle apresentou valores de $b^{*}$ significativamente menores do que os tratamentos contendo óleos vegetais $(\mathrm{p}<0,05)$, uma 
vez que os próprios óleos utilizados apresentam uma coloração mais amarelada quando comparada com a gordura suína.

Uma série de estudos já foi realizada em relação à cor de produtos cárneos cozidos elaborados com óleos vegetais (BISHOP; OLSON; KNIPE, 1993; PANERAS; BLOUKAS, 1994; PANERAS; BLOUKAS; FILIS, 1998; PAPADIMA; BLOUKAS, 1999; CÁCERES; GARCÍA; SELGAS, 2008; CHOI et al., 2009; MORALES-IRIGOYEN et al., 2012). Os resultados encontrados nestes trabalhos mostram que as diferenças estão mais relacionadas ao tipo de produto e as características dos óleos vegetais utilizados. Ainda, a substituição da gordura animal por óleos vegetais aumenta os valores de $L^{*}$ e $b^{*}$, diminuindo os valores de $a^{*}$. Essa relação inversa entre $L^{*}$ e $a^{*}$ também foi observada neste trabalho e está relacionada com a distribuição da fase oleosa na matriz proteica durante a mistura da massa cárnea, uma vez que os óleos vegetais são mais uniformemente dispersos e melhor distribuídos do que a gordura animal, aumentando assim a área das partículas de gordura (YOUSSEF; BARBUT, 2009).

$\mathrm{Na}$ contagem de mesófilos totais, no dia 0 , o maior valor foi encontrado no tratamento $\mathrm{S} 1$, com 3,21 ciclos logaritmos, sendo estatisticamente superior aos demais tratamentos. Ainda, houve crescimento das bactérias aeróbias mesófilas em todos os tratamentos durante o armazenamento em temperatura ambiente. Aos 60 dias de armazenamento, as contagens de bactérias aeróbias mesófilas ficaram entre 6,08 e 7,73 ciclos logaritmos
(Tabela 4), sendo o tratamento controle superior aos demais $(\mathrm{p}<0,05)$. Comportamento similar foi observado nas contagens de bactérias láticas. No dia 0 , apenas no tratamento $\mathrm{C} 1$ foi evidenciada contagens de 1 ciclo logaritmo. No entanto, também houve crescimento das bactérias durante o armazenamento, com valores variando entre 3,41 e 6,09 ciclos logaritmos aos 30 dias de armazenamento e entre 6,46 e 7,69 ciclos logaritmos aos 60 dias de armazenamento. As contagens de bactérias láticas explicam também a queda no $\mathrm{pH}$ observada em todos os tratamentos durante o armazenamento (Tabela 2). A combinação de $\mathrm{pH}$ em torno de 6,0 juntamente com o processamento térmico se mostrou suficiente por produzir mortadelas seguras por pelo menos 30 dias de armazenamento. Vale ressaltar, que os produtos foram armazenados em temperatura ambiente e uma alternativa para aumentar a vida de prateleira seria o armazenamento em temperatura de refrigeração. Os valores encontrados aos 60 dias mostram o início de um processo de deterioração, principalmente com prováveis alterações sensoriais provocadas pela queda do $\mathrm{pH}$.

Em relação à oxidação das gorduras pelo índice de TBARS (Tabela 5), foi possível observar que no dia 0 , os valores variaram entre 0,031 a 0,232 $\mathrm{mg}$ de malonaldeído/kg, sendo o tratamento $\mathrm{S} 1$ o que apresentou o menor valor. Os tratamentos $\mathrm{P}, \mathrm{C} 1, \mathrm{C} 2, \mathrm{~L} 1, \mathrm{O} 1$ e $\mathrm{S} 2$ apresentaram os maiores resultados de TBARS, mas, no entanto, não diferiram estatisticamente entre si. Neste caso, também não foi possível observar uma relação entre os fatores (tipo de óleo e/ou índice de substituição) que influenciasse nos valores de TBARS. 
Tabela 4. Contagem de bactérias aeróbias mesófilas e bactérias láticas em mortadelas elaboradas com diferentes óleos vegetais em dois níveis de substituição da gordura suína (em log UFC. $\left.\mathrm{g}^{-1}\right)$.

\begin{tabular}{ccccccc}
\hline \multirow{2}{*}{ Tratamentos } & \multicolumn{3}{c}{ Bactérias aeróbias mesófilas } & \multicolumn{3}{c}{ Bactérias láticas } \\
\cline { 2 - 7 } & Dia 0 & Dia 30 & Dia 60 & Dia 0 & Dia 30 & Dia 60 \\
\hline P & $2,11 \pm 0,08^{\mathrm{b}}$ & $2,10 \pm 0,08^{\mathrm{a}}$ & $7,73 \pm 0,01^{\mathrm{a}}$ & $<1,0$ & $5,43 \pm 0,05^{\mathrm{c}}$ & $7,12 \pm 0,05^{\mathrm{cd}}$ \\
$\mathrm{C} 1$ & $2,11 \pm 0,09^{\mathrm{b}}$ & $4,37 \pm 0,03^{\mathrm{e}}$ & $6,12 \pm 0,03^{\mathrm{e}}$ & 1,0 & $3,41 \pm 0,05^{\mathrm{f}}$ & $7,69 \pm 0,02^{\mathrm{a}}$ \\
$\mathrm{C} 2$ & $2,14 \pm 0,09^{\mathrm{b}}$ & $4,43 \pm 0,03^{\mathrm{e}}$ & $6,08 \pm 0,06^{\mathrm{e}}$ & $<1,0$ & $4,42 \pm 0,07^{\mathrm{e}}$ & $7,11 \pm 0,07^{\mathrm{d}}$ \\
$\mathrm{L} 1$ & $2,06 \pm 0,05^{\mathrm{b}}$ & $4,84 \pm 0,05^{\mathrm{d}}$ & $6,55 \pm 0,04^{\mathrm{d}}$ & $<1,0$ & $5,41 \pm 0,08^{\mathrm{c}}$ & $7,22 \pm 0,06^{\mathrm{c}}$ \\
L2 & $2,14 \pm 0,11^{\mathrm{b}}$ & $5,40 \pm 0,04^{\mathrm{c}}$ & $6,92 \pm 0,01^{\mathrm{c}}$ & $<1,0$ & $5,39 \pm 0,03^{\mathrm{c}}$ & $7,07 \pm 0,06^{\mathrm{d}}$ \\
O1 & $2,09 \pm 0,09^{\mathrm{b}}$ & $6,19 \pm 0,07^{\mathrm{a}}$ & $7,70 \pm 0,02^{\mathrm{a}}$ & $<1,0$ & $6,09 \pm 0,08^{\mathrm{a}}$ & $7,56 \pm 0,03^{\mathrm{b}}$ \\
O2 & $2,07 \pm 0,06^{\mathrm{b}}$ & $5,75 \pm 0,02^{\mathrm{b}}$ & $6,06 \pm 0,06^{\mathrm{e}}$ & $<1,0$ & $5,86 \pm 0,03^{\mathrm{b}}$ & $6,46 \pm 0,02^{\mathrm{f}}$ \\
S1 & $3,21 \pm 0,04^{\mathrm{a}}$ & $6,23 \pm 0,02^{\mathrm{a}}$ & $7,53 \pm 0,04^{\mathrm{b}}$ & $<1,0$ & $5,80 \pm 0,02^{\mathrm{b}}$ & $7,69 \pm 0,05^{\mathrm{a}}$ \\
S2 & $2,15 \pm 0,10^{\mathrm{b}}$ & $3,95 \pm 0,03^{\mathrm{f}}$ & $6,87 \pm 0,01^{\mathrm{c}}$ & $<1,0$ & $4,57 \pm 0,02^{\mathrm{d}}$ & $6,68 \pm 0,03^{\mathrm{e}}$ \\
\hline
\end{tabular}

${ }^{a}$ Médias acompanhadas pela mesma letra na mesma coluna, não apresentam diferença significativa pelo teste de Tukey.

P - 100\% de gordura suína; C1 - 50\% de óleo de canola; C2 - 100\% de óleo de canola; L1 - 50\% de óleo de linhaça; L2 - 100\% de óleo de linhaça; O1 - 50\% de óleo de oliva; O2 - 100\% de óleo de oliva; S1 - 50\% de óleo de soja; S2 - 100\% de óleo de soja.

Fonte: Elaboração dos autores.

Tabela 5. Valores de TBARS de mortadelas elaboradas com diferentes óleos vegetais em dois níveis de substituição da gordura suína (em mg de malonaldeído/kg).

\begin{tabular}{cccc}
\hline Tratamentos & Dia 0 & Dia 30 & Dia 60 \\
\hline P & $0,224 \pm 0,052^{\mathrm{a}}$ & $0,122 \pm 0,033^{\mathrm{ab}}$ & $0,140 \pm 0,028^{\mathrm{a}}$ \\
C1 & $0,214 \pm 0,018^{\mathrm{ab}}$ & $0,074 \pm 0,027^{\mathrm{ab}}$ & $0,184 \pm 0,051^{\mathrm{a}}$ \\
C2 & $0,232 \pm 0,032^{\mathrm{a}}$ & $0,134 \pm 0,037^{\mathrm{a}}$ & $0,179 \pm 0,007^{\mathrm{a}}$ \\
L1 & $0,158 \pm 0,019^{\mathrm{abc}}$ & $0,063 \pm 0,018^{\mathrm{b}}$ & $0,182 \pm 0,016^{\mathrm{a}}$ \\
L2 & $0,138 \pm 0,016^{\mathrm{bc}}$ & $0,080 \pm 0,023^{\mathrm{ab}}$ & $0,143 \pm 0,027^{\mathrm{a}}$ \\
O1 & $0,158 \pm 0,038^{\mathrm{abc}}$ & $0,105 \pm 0,022^{\mathrm{ab}}$ & $0,187 \pm 0,023^{\mathrm{a}}$ \\
O2 & $0,115 \pm 0,066^{\mathrm{cd}}$ & $0,124 \pm 0,023^{\mathrm{ab}}$ & $0,171 \pm 0,046^{\mathrm{a}}$ \\
S1 & $0,031 \pm 0,012^{\mathrm{d}}$ & $0,078 \pm 0,027^{\mathrm{ab}}$ & $0,210 \pm 0,020^{\mathrm{a}}$ \\
S2 & $0,152 \pm 0,018^{\mathrm{abc}}$ & $0,101 \pm 0,021^{\mathrm{ab}}$ & $0,195 \pm 0,047^{\mathrm{a}}$ \\
\hline
\end{tabular}

${ }^{a}$ Médias acompanhadas pela mesma letra na mesma coluna, não apresentam diferença significativa pelo teste de Tukey. P-100\% de gordura suína; $\mathrm{C} 1-50 \%$ de óleo de canola; $\mathrm{C} 2-100 \%$ de óleo de canola; L1 - 50\% de óleo de linhaça; L2 - $100 \%$ de óleo de linhaça; O1 - 50\% de óleo de oliva; O2 - 100\% de óleo de oliva; S1 - 50\% de óleo de soja; S2 - 100\% de óleo de soja. Fonte: Elaboração dos autores.

No entanto, houve uma redução nos valores de TBARS aos 30 dias de armazenamento para alguns tratamentos, com valores variando entre 0,063 e $0,134 \mathrm{mg}$ de malonaldeído/kg. Foi possível observar diferença significativa entre o tratamento $\mathrm{C} 2$ e o L1, que apresentaram o maior e o menor valor de TBARS, respectivamente. Porém, não houve diferença significativa entre estes tratamentos e os demais. Essa redução nos valores de TBARS pode ser atribuída principalmente por uma decomposição do malonaldeído por alguns tipos de microrganismos
(SMITH; ALFORD, 1968; MOERCK; BALL, 1974) ou por oxidação do malonaldeído por outros produtos, como alcoóis e ácidos que não reagem com o ácido tiobarbitúrico (FERNÁNDEZ; PÉREZÁLVAREZ; FERNÁNDEZ-LÓPEZ, 1997). Aos 60 dias de armazenamento, foi observado um aumento nos valores de TBARS em todos os tratamentos. Os valores variaram entre 0,140 e $0,210 \mathrm{mg}$ de malonaldeído/kg, não havendo diferenças significativas entre os tratamentos. Vale ressaltar que todos os resultados encontrados estão de acordo 
com Gray e Pearson (1987), que destacam o valor de $0,5 \mathrm{mg} / \mathrm{kg}$ de malonaldeído como o limiar em que os consumidores conseguiriam detectar a rancidez através de parâmetros organolépticos. A boa estabilidade oxidativa encontrada neste trabalho pode ser atribuída aos óleos vegetais possuírem antioxidantes naturais como isoflavonas, polifenóis e tocoferóis (VISIOLI; POLI; GALLI, 2002; VERNAZA et al., 2012), além da qualidade das embalagens utilizadas, que possui baixa permeabilidade ao oxigênio.

Os valores de TBARS encontrados neste trabalho estão de acordo com os demonstrados por Sheard et al. (2000) e Valencia et al. (2008), que não encontraram diferenças estatísticas nos valores de TBARS tanto em produtos feitos com a carne de animais em que a alimentação foi suplementada com óleos vegetais quanto em produtos em que a gordura animal foi substituída pelos óleos. No entanto, Kayaardi e Gök (2003) e Pelser et al. (2007) relataram valores de TBARS superiores ao controle em embutidos fermentados contendo óleos vegetais. Ainda, Muguerza, Ansorena e Astiasarán (2003) demonstraram que a substituição da gordura suína por óleo de soja em Chorizo de Pamplona foi capaz de reduzir os valores de TBARS.

Nas micrografias (Figura 1) é possível observar uma diferença na quantidade de gordura (identificada como estruturas mais escuras na figura) do tratamento controle (A) em relação aos tratamentos C2 (B), L2 (C), O2 (D) e S2 (E). Considerando que o teor de gordura encontrado nas mortadelas foi de 16,$19 ; 12,72 ; 15,46 ; 12,51$ e 14,96\%, respectivamente, pode-se perceber uma relação com o teor de gordura evidenciado pela micrografia com o resultado encontrado nas análises laboratoriais. As micrografias dos produtos estão muito próximas das descritas por Carballo et al. (1996) em produto cárneo cozido e apresentam coesão e um pouco de proteína granular na matriz cárnea, como também foi observado por Andrés, Zaritzky e Califano (2009). Também foi possível observar uma estrutura esponjosa nos produtos, com espaços onde as gotículas de gordura estão localizadas na matriz cárnea e poros maiores nos tratamentos contendo os óleos vegetais.

Os resultados do presente estudo demonstraram que a substituição parcial ou total da gordura animal pelos óleos vegetais de canola, linhaça, oliva ou soja é uma alternativa viável para a produção de mortadela. Os produtos apresentaram características satisfatórias observando-se as variáveis de qualidade, estabilidade oxidativa e de microestrutura de mortadela durante o período de armazenamento estudado. Todos os parâmetros físico-químicos mostraram-se dentro dos exigidos pela legislação. No entanto, a substituição da gordura animal por óleos vegetais afetou os parâmetros de cor instrumental $\left(L^{*}, a^{*}\right.$ e $\left.b^{*}\right)$. As mortadelas apresentaram crescimento microbiano previsível com o produto e sua forma de armazenamento e boa estabilidade oxidativa, com os valores de TBARS permanecendo abaixo do limiar detectável pelo consumidor durante o período de armazenamento. 
Figura 1. Micrografias das mortadelas elaboradas com diferentes óleos vegetais em de substituição da gordura suína.
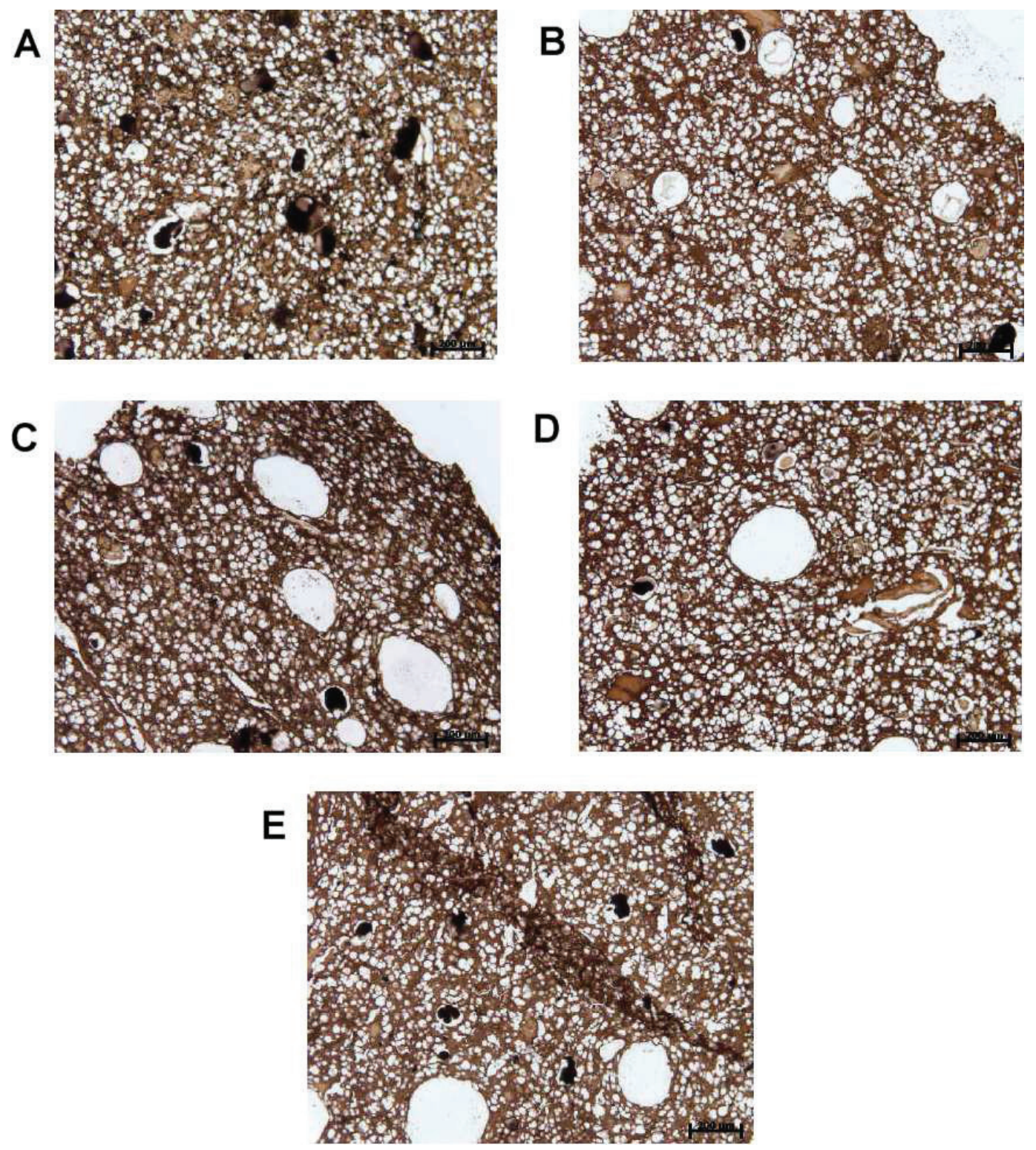

(A) Tratamento Controle - 100\% de gordura suína; (B) Tratamento C2 - 100\% de óleo de canola; (C) Tratamento L2 - 100\% de óleo de linhaça; (D) Tratamento O2 - 100\% de óleo de oliva; (E) Tratamento S2 - 100\% de óleo de soja. Fonte: Elaboração dos autores.

\section{Referências}

ALESON-CARBONELL，L.; FERNÁNDEZ-GINÉZ, J. M.; FERNÁNDES-LÓPEZ, J.; SAYAS-BARBERÁ, M.; PÉREZ-ÁLVAREZ, J. A. La fibra dietetica en la alimentación. Alimentación Equipos y Tecnología, Madrid, v. 21, n. 169, p. 83-91, 2002.

ANDRÉS, S. C.; ZARITZKY, N. E.; CALIFANO, A. $\mathrm{N}$. Innovations in the development of healthier chicken sausages formulated with different lipid sources. Poultry Science, Champaign, v. 88, n. 8, p. 1755-1764, 2009.

ARIHARA, K. Strategies for designing novel functional meat products. Meat Science, Barking, v. 74, n. 1, p. 219229, 2006.

BANCROFT, J. D.; GAMBLE, M. Theory and practice of histological techniques. 5. ed. Edinburgh, London: Churchill Livingstone, 2002. 640 p. 
BARBUT, S.; MITTAL, G. S. Physical and sensory properties of reduced fat breakfast sausages. Journal of Muscle Foods, Hoboken, v. 6, n. 1, p. 47-62, 1995.

BISHOP, D. J.; OLSON, D. G.; KNIPE, C. L. Preemulsified corn oil, pork fat, or added moisture affect quality of reduced fat bologna quality. Journal of Food Science, Chicago, v. 58, n. 3, p.484-487, 1993.

BLOUKAS, J. G.; PANERAS, E. D. Substituting olive oil pork back fat affect quality of low-fat frankfurters. Journal of Food Science, Chicago, v. 58, n. 4, p. 705709, 1993.

BRASIL. Ministério da Agricultura, do Abastecimento e da Reforma Agrária. Secretaria de Defesa Agropecuária. Instrução Normativa $n^{\circ} .4$, de 31/03/2000, Regulamentos Técnicos de Identidade e Qualidade de Carne Mecanicamente Separada, de Mortadela, de Linguiça e de Salsicha. Diário Oficial [da] República Federativa do Brasil, Brasília, 5 de abril 2000. Seção I, p. 6-10.

Secretaria de Defesa Agropecuária. Instrução Normativa $n^{\circ} .62$ de 26/08/2003. Oficializa os métodos analíticos oficiais para análises microbiológicas para controle de produtos de origem animal e água. Diário Oficial [da] Repúbica Federativa do Brasil, Brasília, 18 de setembro. 2003. Seção I, p. 14.

CÁCERES, E.; GARCÍA, M. L.; SELGAS, M. D. Design of a new cooked meat sausage enriched with calcium. Meat Science, Barking, v. 73, n. 2, p. 368-377, 2006.

CÁCERES, E.; GARCÍA, M. L.; SELGAS, M. D. Effect of pre-emulsified fish oil - as source of PUFA n-3 - on microstructure and sensory properties of mortadella, a Spanish bologna-type sausage. Meat Science, Barking, v. 80, n. 2, p. 183-193, 2008.

CÁCERES, E.; GARCÍA, M. L.; TORO, J.; SELGAS, M. D. The effect of fructooligosaccharides on the sensory characteristics of cooked sausages. Meat Science, Barking, v. 68, n. 1, p. 87-96, 2004.

CARBALLO, J.; FERNÁNDEZ, P.; BARRETO, G.; SOLAS, M. T.; JIMENEZ-COLMENERO, F. Morphology and texture of bologna sausage as related to content of fat, starch and egg white. Journal of Food Science, Chicago, v. 61, n. 3, p. 652-655, 1996.

CHOI, Y. S.; CHOI, J. H.; HAN, D. J.; KIM, H. Y.; LEE, M. A.; KIM, H. W.; JEONG, J. Y. KIM, C. J. Characteristics of low-fat meat emulsion systems with pork fat replaced by vegetable oils and rice bran fiber. Meat Science, Barking, v. 82, n. 2, p. 266-271, 2009.

CLAUS, J. R.; HUNT, M. C. Low fat, high added-water bologna sausages formulated with texture modifying ingredients. Journal of Food Science, Chicago, v. 56, n. 3, p. 643-647, 1991.
CLAUS, J. R.; HUNT, M. C.; KASTNER, C. L. Effects of substituting addes water for fat on the textural sensory and processing characteristics of bologna. Journal of Muscle Foods, Hoboken, n. 1, p. 1-12, 1989.

DEL NOBILE, M. A.; CONTE, A.; INCORONATO, A. L.; PANZA, O.; SEVI, A.; MARINO, R. New strategies for reducing pork back-fat content in typical Italian salami. Meat Science, Barking, v. 81, n. 1, p. 253-269, 2009.

FERNÁNDEZ, J.; PÉREZ-ÁLVAREZ, J. A.; FERNÁNDEZ-LÓPEZ, J. A. Thiobarbituric acid test for monitoring lipid oxidation in meat. Food Chemistry, Oxford, v. 59, n. 3, p. 345-353, 1997.

FERNÁNDEZ-GINÉS, J. M.; FERNÁNDEZ-LÓPEZ, J.; SAYAS-BARBERÁ, E.; PÉREZ-ALVAREZ, J. Meat products as functional foods: A review. Journal of Food Science, Chicago, v. 70, n. 2, p. R37-R43, 2005.

FERNÁNDEZ-GINÉS, J. M.; FERNÁNDEZ-LÓPEZ, J.; SAYAS-BARBERÁ, E.; SENDRA, E.; PÉREZALVAREZ, J. A. Effect of storage conditions on quality characteristics of Bologna sausages made with citrus fibre. Journal of Food Science, Chicago, v. 68, n. 2, p. 710-715, 2003.

GRAY, J. I.; PEARSON, A. M. Rancidity and warmedover flavor. In: PEARSON, A. M.; DUTSON, T. R. Advances in meat research, Vol.3., restructured meat and poultry products. New York: Van Nostrand Reinhold Co., 1987. p. 221-269.

GRIGELMO-MIGUEL, N.; ABADÍAS-SERÓS, M. I.; MARTÍN-BELLOSO, O. Characterisation of low-fat high-dietary fiber frankfurters. Meat Science, Barking, v. 52, n. 3, p. 247-256, 1999.

HOZ, L.; D'ARRIGO, M.; CAMBERO, I.; ORDÓÑEZ, J. A. Development of an n-3 fatty acid and $\alpha$-tocopherol enriched dry fermented sausage. Meat Science, Barking, v. 67, n. 3, p. 485-495, 2004.

JIMÉNEZ-COLMENERO, F. Healthier lipid formulation approaches in meat-based functional foods. Technological options for replacement of meat fats by non-meat fats. Trends in Food Science \& Technology, Amsterdam, v. 18, n. 11, p. 567-578, 2007.

JIMÉNEZ-COLMENERO, F.; REIG, M.; TOLDRÁ, F. New approaches for the development of functional meat products. In: NOLLET, L. M. L.; TOLDRÁ, F. Advanced technologies for meat processing. London, New York: Boca Raton, 2006, p. 275-308.

KAYAARDI, S.; GOK, V. Effect of replacing beef fat with olive oil on quality characteristics of Turkish Soudjouk (Sucuk). Meat Science, Barking, v. 66, n. 1, p. 249-257, 2003. 
LINDAHL, G.; LUNDSTRÖM, K.; TORNBERG, E. Contribution of pigment content, myoglobin forms and internal reflectance to the lightness of pork loin and ham from pure breed pigs. Meat Science, Barking, v. 59, n. 2, p. 141-151, 2001.

LURUEÑA-MARTÍNEZ, M. A.; VIVAR-QUINTANA, A. M.; REVILLA, I. Effect of locust bean/xanthan gum addition and replacement of pork fat with olive oil on the quality characteristics of low-fat frankfurters. Meat Science, Barking, v. 68, n. 3, p. 383-389, 2004.

MACDOUGALL, D. B. Colour measurement of food: Principles and practice. In: (Ed.). Colour in food. Cambridge, UK: Woodhead Publishing in Food Science and Technology, 2000. p. 33-63.

MOERCK, K. E.; BALL, H. R. Lipid oxidation in mechanically deboned chicken meat. Journal of Food Science, Chicago, v. 39, p. 876-879, 1974.

MORALES-IRIGOYEN, E. E.; SEVERIANO-PÉREZ, P.; RODRIGUEZ-HUEZO, M. E.; TOTOSAUS, A. Textural, physicochemical and sensory properties compensation of fat replacing in pork liver pâté incorporating emulsified canola oil. Food Science and Technology International, Madrid, v. 18, n. 4, p. 413-422, 2012.

MUGUERZA, E.; GIMENO, O.; ANSORENA, D.; BLOUKAS, J. G.; ASTIASARAN, I. Effect of replacing pork backfat with pre-emulsified olive oil on lipid fraction and sensory quality of Chorizo de Pamplona a traditional Spanish fermented sausage. Meat Science, Barking, v. 59, n. 3, p. 251-258, 2001.

MUGUERZA, E.; ANSORENA, D.; ASTIASARÁN, I. Functional dry fermented sausages manufactured with high levels of n-3 fatty acids: nutritional benefits and evaluation of oxidation. Journal of the Science of Food and Agriculture, London, v. 84, n. 9, p. 1061-1068, 2004.

MUGUERZA, E.; ANSORENA, D.; ASTIASARÁN, I. Improvement of nutritional properties of Chorizo de Pamplona by replacement of pork backfat with soy oil. Meat Science, Barking, v. 65, n. 4, p. 1361-1367, 2003.

MUGUERZA, E.; FISTA, G.; ANSORENA, D.; ASTIASARAN, I.; BLOUKAS, J. G. Effect of fat level and partial replacement of pork backfat with olive oil and processing and quality characteristics of fermented sausages. Meat Science, Barking, v. 61, n. 4, p. 397-404, 2002 .

PANERAS, E. D.; BLOUKAS, J. G. Vegetable oils replace pork backfat for low-fat frankfurters. Journal of Food Science, Chicago, v. 59, n. 4, p. 725-733, 1994.
PANERAS, E. D.; BLOUKAS, J. G.; PAPADIMA, S. N. Effect of meat source and fat level on processing and quality characteristics of frankfurters. $L W T-$ Food Science and Technology, Oxford, v. 29, n. 5-6, p. 507514, 1996.

PANERAS, E. D; BLOUKAS, J. G.; FILIS, D. G. Production of low-fat frankfurters with vegetable oils following the dietary guidelines for fatty acids. Journal of Muscle Foods, Hoboken, v. 9, n. 2, p. 111-126, 1998.

PAPADIMA, S. N.; BLOUKAS, J. G. Effect of the fat level and storage conditions on quality characteristics on traditional Greek sausages. Meat Science, Barking, v. 51, n. 2, p. 103-113, 1999.

PELSER, W. M.; LINSSEN, J. P.; LEGGER, A.; HOUBEN, J. H. Lipid oxidation in n-3 fatty acid enriched Dutch style fermented sausages. Meat Science, Barking, v. 75, n. 1, p. 1-11, 2007.

RAHARJO, S.; SOFOS, J. N.; SCHMIDT, G. R. Improved speed, specifity, and limit of determination of an aqueous acid extraction thiobarbituric acid $-\mathrm{C}_{18}$ method for measuring lipid peroxidation in beef. Journal of Agricultural and Food Chemistry, Washington, v. 40, n. 11, p. 2182-2185, 1992.

SANTOS, C.; CAMBERO, M. I.; CABEZA, M. C.; ORDÓÑEZ, J. A. Enrichment of dry-cured ham with $\alpha$-linolenic acid and $\alpha$-tocopherol by the use of linseed oil and $\alpha$-tocopheryl acetate in pig diets. Meat Science, Barking, v. 80, n. 2, p. 668-674, 2008.

SHEARD, P. R.; ENSER, M.; WOOD, J. D.; NUTE, G. R.; GILL, B. P.; RICHARDSON, R. I. Shelf life and quality of pork products with raised n-3 PUFA. Meat Science, Barking, v. 55, n. 2, p. 213-221, 2000.

SINGH, R.; CHATLI, M. K.; BISWAS, A. K.; SAHOO, J. Quality and storage stability of chicken meat patties incorporated with linseed oil. Journal of Food Quality, Malden, v. 34, n. 5, p. 352-362, 2011.

SMITH, J. L.; ALFORD, J. A. Action of microorganisms on the peroxides and carbonyls of rancid fat. Journal of Food Science, Chicago, v. 33, n. 1, p. 93-97, 1968.

TERRA, N. N. Apontamentos de tecnologia de carnes. São Leopoldo: Unisinos, 1998. 216 p.

TERRA, N. N.; BRUM, M. A. R. Carne e seus derivados - técnicas de controle de qualidade. São Paulo: Nobel, 1988. 119 p.

TROUTT, E. S.; HUNT, M. C.; JOHNSON, D. E.; CLAUS, J. R.; KASTNER, C. L.; KROPF, D. H. Characteristics of low-fat ground beef containing texture-modifying ingredients. Journal of Food Science, Chicago, v. 57, n. 1, p. 19-24, 1992. 
VALENCIA, I.; O'GRADY, M. N.; ANSORENA, D.; ASTIASARÁN, I.; KERRY, J. P. Enhancement of the nutritional status and quality of fresh pork sausages following the addition of linseed oil, fish oil and natural antioxidants. Meat Science, Barking, v. 80, n. 4, p. 10461054, 2008.

VERNAZA, M. G.; DIA, V. P.; MEJIA, E. G.; CHANG, Y. K. Antioxidant and antiinflammatory propertied of germinated and hydrolysed Brazilian soybean flours. Food Chemistry, Oxford, v. 134, n. 4, p. 2217-2225, 2012.

VISIOLI, F.; POLI, A.; GALLI, C. Antioxidant and other biological activities of phenols from olives and olive oil. Medical Care Research Review, Thousand Oaks, v. 22, n. 1, p. 65-75, 2002.
VIUDA-MARTOS, M.; RUIZ-NAVAJAS, Y.; FERNÁNDEZ-LÓPEZ, J.; PÉREZ-ÁLVAREZ, J. A. Effect of added citrus fibre and spice essential oils on quality characteristics and shelf-life of mortadella. Meat Science, Barking, v. 85, n. 3, p. 568-576, 2010.

YOUSSEF, M. K.; BARBUT, S. Effects of protein level and fat/oil on emulsion stability, texture, microstructure and color of meat batters. Meat Science, Barking, v. 82, n. 2, p. 228-233, 2009.

Fat reduction in comminuted meat productseffects of beef fat, regular and pre-emulsified canola oil. Meat Science, Barking, v. 87, n. 4, p. 356-360, 2011. 